Ann. Zootech., I968, 17 (4), 409-427.

\title{
VALEUR ALIMENTAIRE DES PROTÉINES DE LA LEVURE SULFITIQUE ET DU LACTOSÉRUM LEVURÉ CHEZ LE PORC EN CROISSANCE
}

\author{
F. COLOMER-ROCHER, C. FÉVRIER \\ avec la collaboration technique de A. GAYE \\ Station de Recherches sur l'Élevage des Porcs, \\ Centre national de Recherches zootechniques, 78 -Jouy-en-Josas \\ Institut national de la Recherche agronomique
}

\section{SOMMAIRE}

Au cours d'une expérience portant sur 3 lots de 3 porcs entre 3 o et go $\mathrm{kg}$ de poids vif, on a comparé la valeur nutritive des protéines de la levure sulfitique (cultivée sur résidus de pâtes à papier), du lactosérum "levuré " et du tourteau de soja, en supplémentation d'un régime à base d'orge. Les régimes utilisés étaient isoprotéiques, isoénergétiques et isocellulosiques. Le rationnement en fonction de l'âge a été identicue pour tous les animaux et ajusté sur la consommation minimum observée.

L.es résultats obtenus montrent que les coefficients d'utilisation digestive de la matière organique et de l'azote sont, en moyenne, comparables entre les trois régimes. Par contre, la quantité d'azote retenue par jour avec le régime orge-soja est significativement supérieure à celle retenue avec le régime orge-levure sulfitique et orge lactosérum levuré. Corrélativement, le coefficient de rétention de l'azote du régime orge-soja $\left(5^{5}, 4 \mathrm{p}\right.$. гоo) est significativement plus élevé que celui obtenu avec le régime orge-levure sulfitique $(44,2 \mathrm{p}$. Ioo) ou avec le régime orge-lactosérum levuré $(44,5 \mathrm{p}$. Ioo). Ces résultats sont confirmés par ceux de la découpe. Les animaux recevant le régime orge-soja sont plus maigres que ceux des deux autres régimes.

Ces résultats suggèrent que dans un régime à base d'orgc, les protéines du soja sont mieux adaptées à couvrir les besoins des porcs en acides aminés entre 30 et $90 \mathrm{~kg}$ de poids vif, que celles des produits " levurés " employés. Ceci est en accord avec la composition en acides aminés du régime orgelevure sulfitique ; cependant, il y a une contradiction pour le régime orge-lactosérum levuré qui, selon les analyses, est plus riche en acides aminés essentiels que le régime orge-soja. Dans ce dernier cas, on peut émettre l'hýpothèse d'une indisponibilité de la lysine dans le lactosérum levuré due au traitement industriel.

\section{INTRODUCTION}

On sait depuis longtemps que les levures peuvent être cultivées sur toutes sortes de milieux nutritifs riches en glucides, et qu'elles peuvent synthétiser leurs protéines à partir de l'azote inorganique ajouté aux milieux de culture. 
Cependant, la production de levures est encore faible, aussi bien en regard des besoins immenses en protéines sur le plan mondial, que vis-à-vis des capacités théoriques d'obtention à partir des sous-produits des industries agricoles, alimentaires et pétrolières.

Le traitement de certains de ces résidus industriels par les levures permet une augmentation de leur teneur en protéines. Ainsi, I ooo 1 de lactosérum contiennent en moyenne $60 \mathrm{~kg}$ de matière sèche avec une teneur de I2,5 p. Ioo de protéines, soit $7,5 \mathrm{~kg}$ de protéines brutes. Le traitement par la levure permet d'obtenir $40 \mathrm{~kg} \mathrm{de}$ matière sèche à $30 \mathrm{p}$. Ioo de protéines, soit I2 $\mathrm{kg}$ de protéines brutes (NAIDITCH, I96I).

Le traitement par la levure de lessives sulfitiques, résiduaires de la fabrication de la pâte à papier, permet un rendement industriel de 35 p. Ioo (exprimé en levure ensachée par rapport aux sucres totaux mis en œuvre). I, a teneur en protéines du produit fini est de $50 \mathrm{p}$. IOO (LEFRANçors et REVUZ, I964).

Cependant, si l'augmentation de la teneur totale en protéines est évidente, il est nécessaire de connaître la valeur nutritionnelle des produits obtenus. Or, la valeur d'une levure alimentaire dépend, d'une part de la souche utilisée, mais plus encore de la nature du milieu sur lequel elle est cultivée et des conditions d'humidité, de température et de durée du séchage (JACQUOT, I946).

Il y a donc lieu de distinguer les résultats obtenus en fonction de l'origine de la levure, du milieu de culture et du mode de fabrication.

Les études concernant l'utilisation des levures dans l'alimentation du Porc ont porté sur la comparaison de leur valeur nutritive par rapport aux protéines d'origine animale : farine de viande (LEROY et FÉvrIER, I947 ; FÉvrIER et BocC.ARD, I957; Palamaru et al., I966; Ruszczyc et Glaps, I959) ; farine de poisson (Glaps et Ruszczyc, I955) ; lait écrémé (BoKorov et al., I963) ou bien sur les niveaux d'incorporation de levure à des régimes d'engraissement (CsokA, I965) ou encore sur l'appétibilité des différentes sortes de levures chez le porcelet (SALMON-LEGAGNEEUR, Ig60). Cependant, à notre connaissance, il n'existe pas d'expérience portant sur la comparaison de la valeur des levures à celles des protéines d'origine végétale. Or, dans les régimes pour Porc actuellement employés, le tourteau de soja prend une place de plus en plus importante, aussi est-il logique de le comparer à la levure ; d'autant que dans les deux cas, les teneurs en protéines brutes sont comparables et que les acides aminés soufrés constituent leur facteur limitant, avec, pour les levures, une teneur en 1ysine totale plus élevée.

L’expérience que nous avons réalisée porte sur la comparaison de la supplémentation d'un régime à base d'orge par trois sources de protéines différentes, soit le tourteau de soja, soit la levure sulfitique, soit le lactosérum levuré.

La levure sulfitique utilisée est obtenue à partir de la culture de Torula utilis sur résidus de pâtes à papier, milieux dont on isole les levures que l'on sèche ensuite.

Le lactosérum levuré est obtenu par la culture de Torula cremoris, sur lactosérum acide; dans ce cas, les levures sont séchées en même temps que leur milieu de culture. 


\section{MATÉRIEL ET MÉTHODES}

L'expérience porte sur la détermination de la digestibilité des protéines et sur la rétention azotée chez le Porc en croissance recevant l'un des régimes suivants :

$$
\begin{aligned}
& \text { I - Orge-tourteau de soja. } \\
& \text { II - Orge-levure sulfitique. } \\
& \text { III - Orge-lactosérum levuré. }
\end{aligned}
$$

Dans tous les régimes, les protéines de l'orge représentent $52 \mathrm{p}$. 100 des protéines totales, qui constituent 13 p. roo de la ration.

Ce taux de I 3 p. Ioo de protéines a été choisi de manière à fournir journellement une quantité sub-optimale de protéines par rapport aux besoins, afin de mettre en évidence les différences de qualité entre les protéines.

Les régimes sont isoprotéiques, isocellulosiques et isoénergétiques. Enfin, compte tenu de la très grande richesse en minéraux du lactosérum levuré, les taux de calcium, de phosphore et de sodium ont été égalisés entre les trois régimes.

La composition des aliments utilisés est rapportée dans le tableau $\mathrm{I}$ et leur teneur en acides aminés (PION et FAUCONNEAU, I 966 ; PION, 1967) est donnée dans le tableau 2 . La nature des régimes est définie dans le tableau 3 et les résultats d'analyse sont donnés dans le tableau 4.

Dans cette expérience, nous n'avons pas voulu tenir compte de la teneur en vitamines des levures et un mélange vitaminique a été apporté dans tous les régimes, de manière à couvrir largement les besoins des animaux.

\section{Animaux}

L'expérience a porté sur trois lots de trois porcs mâles castrés de race Large White. Chaque série, comprenant une répétition du traitement expérimental, était constituée par trois animaux de même portée et d'âge comparable.

Les porcs ont été maintenus en cage de digestibilité pendant toute la durée de l'expérience et la pesée des animaux a été effectuée chaque semaine.

\section{Rationnement}

Le rationnement, en fonction du temps, a été le même pour tous les animaux dans tous les lots. Pendant chaque période de collecte, les quantités distribuées ont été maintenues constantes et ont été augmentées entre chaque période.

A la fin de la dernière période de collecte, soit vers $83 \mathrm{~kg}$ de poids vif, l'aliment a été fourni ad libitum jusqu'à l'abattage à $90 \mathrm{~kg}$.

\section{Période de collecte}

Les collectes ont été effectuées au cours de 7 périodes de 6 jours. La première de ces périodes a eu lieu deux semaines après la mise en cage des animaux et après leur adaptation aux régimes expérimentaux, soit à un poids moyen de $3 \mathrm{I} \mathrm{kg}$. Les deux premières collectes ont eu lieu à $\mathrm{I}_{4}$ jours d'intervalle et les collectes suivantes à $2 \mathbf{r}$ jours d'intervalle.

La technique utilisée pour l'échantillonnage de l'aliment consommé et des excrétats ainsi que les méthodes d'analyse utilisées au laboratoire ont été décrites précédemment (HENRY et RÉRAT, I966).

\section{Analyse statistique des résultats}

L'analyse statistique des résultats a été faite suivant un schéma en "blocs randomisés" (Cochran et Cox, I957). Il a été tenu compte en outre, de l'effet " période " et des interactions 
F. COLOMER-ROCHER, C. FÉVRIER

TABLEAU I

Composition chimique des aliments en pourcentage

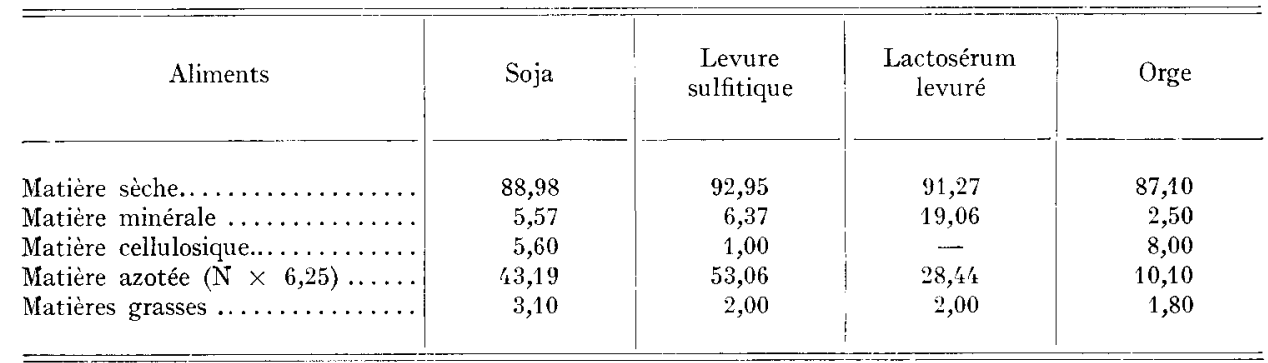

TABLEAU 2

Teneurs en acides aminés (PıN, 1966-1967) (g/16 g N)

\begin{tabular}{|c|c|c|c|c|}
\hline & Soja & $\begin{array}{l}\text { Levure } \\
\text { sulfitique }\end{array}$ & $\begin{array}{l}\text { Lactosérum } \\
\text { levuré }\end{array}$ & $\begin{array}{c}\text { Orge } \\
\text { (moyenne en N) }\end{array}$ \\
\hline 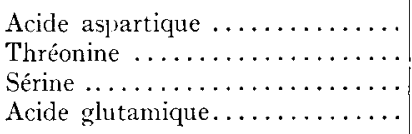 & $\begin{array}{c}11,7 \\
4,05 \\
5,1 \\
18,6\end{array}$ & $\begin{array}{c}7,6 \\
4,2 \\
3,75 \\
12,5\end{array}$ & $\begin{array}{r}9,35 \\
5,7 \\
5,0 \\
14,4\end{array}$ & $\begin{array}{r}5,85 \\
3,45 \\
4,3 \\
23,1\end{array}$ \\
\hline 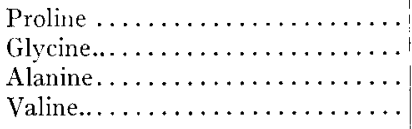 & $\begin{array}{l}5,35 \\
4,4 \\
4,55 \\
5,5\end{array}$ & $\begin{array}{l}3,0 \\
4,05 \\
5,6 \\
5,1\end{array}$ & $\begin{array}{l}4,8 \\
2,85 \\
5,0 \\
5,4\end{array}$ & $\begin{array}{l}10,85 \\
4,2 \\
4,2 \\
5,45\end{array}$ \\
\hline 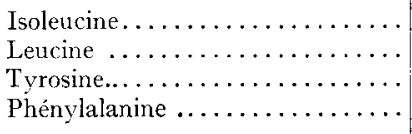 & $\begin{array}{l}5,1 \\
7,9 \\
4,0 \\
5,4\end{array}$ & $\begin{array}{l}4,45 \\
6,3 \\
3,3 \\
3,65\end{array}$ & $\begin{array}{l}5,3 \\
8,6 \\
3,6 \\
3,4\end{array}$ & $\begin{array}{l}3,85 \\
7,0 \\
3,35 \\
5,3\end{array}$ \\
\hline 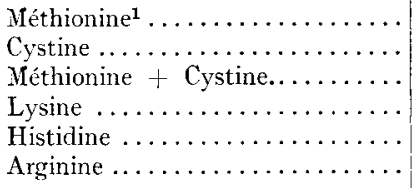 & $\begin{array}{l}1,3 \\
1,6 \\
2,9 \\
6,6 \\
2,75 \\
7,6\end{array}$ & $\begin{array}{l}1,3 \\
0,95 \\
2,25 \\
7,25 \\
2,0 \\
4,75\end{array}$ & $\begin{array}{l}1,65 \\
1,6 \\
3,25 \\
7,65 \\
1,85 \\
3,35\end{array}$ & $\begin{array}{l}1,9 \\
2,5 \\
4,4 \\
3,65 \\
2,1 \\
4,8\end{array}$ \\
\hline Matières azotées p. 100 MS .... & 59 & 57,1 & 31,2 & 11,0 \\
\hline Nombre d'échantillons ......... & 3 & 2 & 2 & 3 \\
\hline
\end{tabular}

(1) Dosée après oxydation en méthionine sulfone. 
TABLEAUX 3 et 4

Composition des régimes en pourcentage

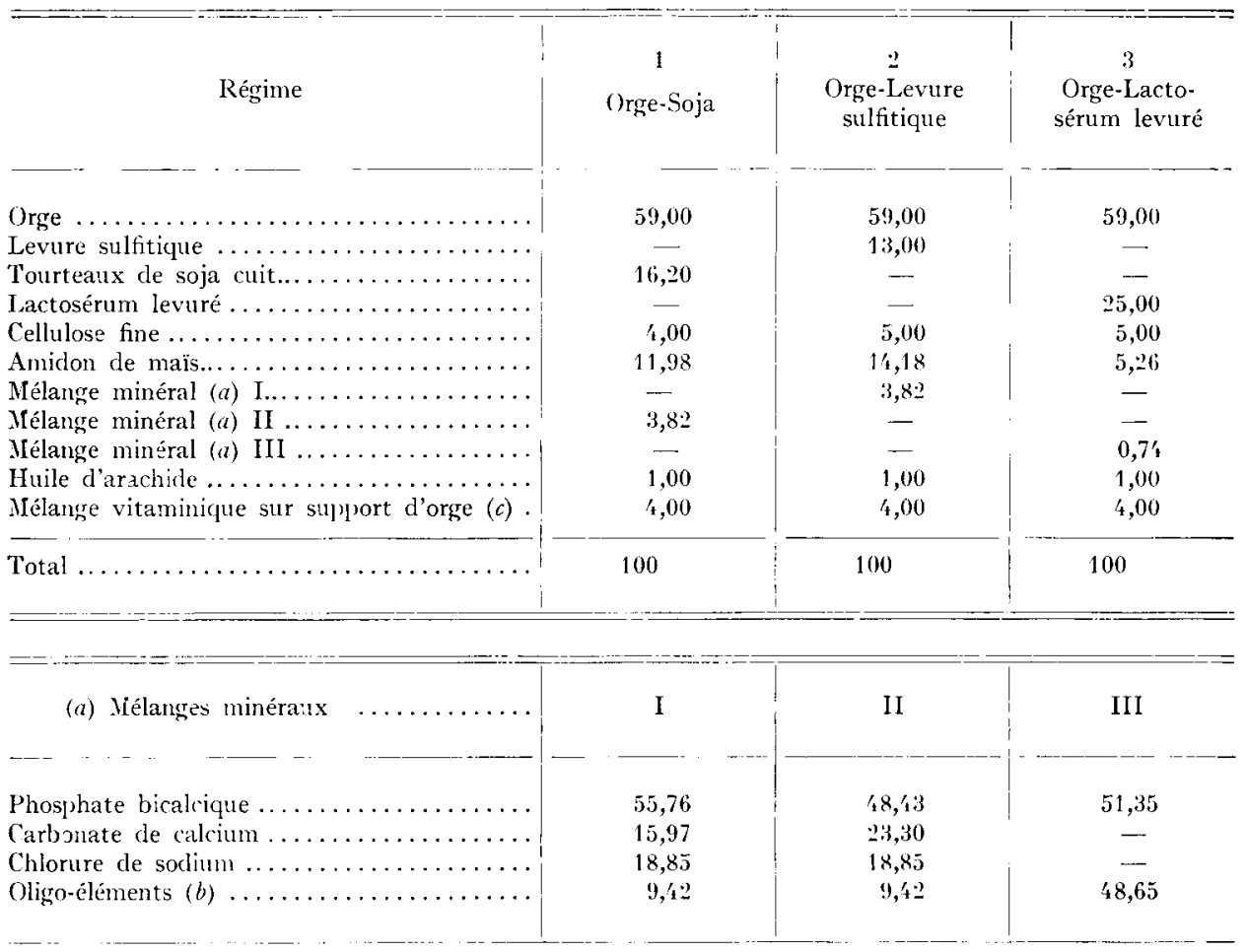

(b) Carbonate de magnésium 8,3,33. Sulfate ferreux 8,33. Sulfate de manganése $2,50$. Sulfate de cuivre $0,8^{\prime}$. Sulfate de zinc ' 16 . Sulfate de cobalt 0,42 . Iodure de potassium 0,12 .

(c) Composition p. $100 \mathrm{k}$ : de régim: : Vit. A 500000 U.I. Vit. D2 50000 U.I. Riboflavine $200 \mathrm{mg}$. Acicle pantothénique $500 \mathrm{mg}$. Choline $100 \mathrm{mg}$. Vit. $3_{12} 1 \mathrm{mg}$.

Composition chimique des régimes

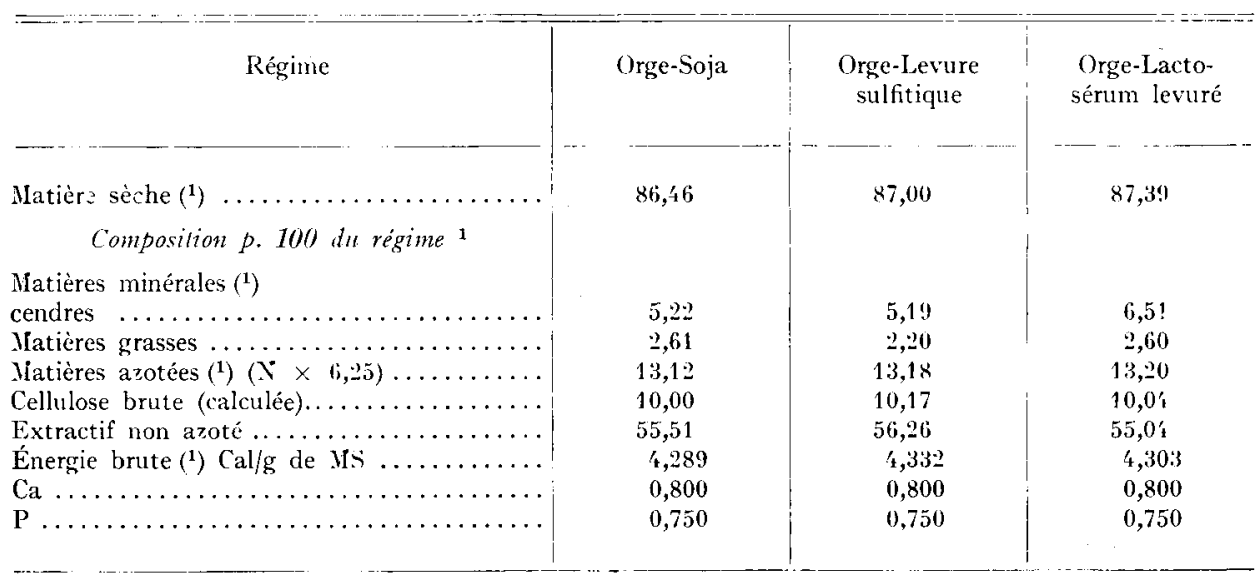

(1) Valeur moyenne sur l'ensemble de sept périodes.

Annales de Zootechnie. - 1968. 
" périodes $\times$ traitement ". Ces effets dont la décomposition est détaillée ci-après sont testés par rapport à une résiduelle entre-animaux ou intra-animal.

Source de variations

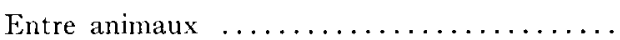

- Répétitions .........................

- Régimes .........................

- Résiduelle entre animaux...............

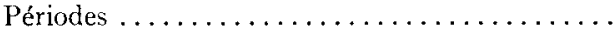

Interactions périodes $\times$ régimes...............

Résiduelle entre périodes ou intra-animal .....

Variation totale .....................

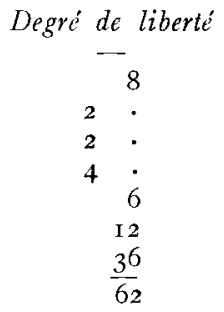

\section{RÉSULTATS}

\section{Consommation d'aliment et vitesse de croissance}

La consommation d'aliment imposée est restée faible tout au long de l'expérience puisqu'elle ne dépassait pas $2 \mathrm{~kg}$ par tête et par jour ; les vitesses de croissance obtenues sont, par conséquent, également faibles (tabl. 5).

TABLEAU 5

Rationnement et gain de poids moyen quotidien

(G. M. Q.)

\begin{tabular}{|c|c|c|c|c|c|c|c|c|}
\hline Période & 1 & 2 & 3 & 4 & 5 & 6 & 7 & Moyenne \\
\hline Rationnement $\mathrm{g} /$ jour & 900 & 1000 & 1400 & 1700 & 1900 & $\simeq 000$ & $\because 000$ & 1571 \\
\hline $\begin{array}{l}\text { I. Orge-Soja : } \\
\text { Poids moyen } \mathrm{kg} . \\
\text { G.ki.O. en } \mathrm{g} . . .\end{array}$ & $\begin{array}{l}31, \mathrm{i} \\
195\end{array}$ & $\begin{array}{l}36,1 \\
361\end{array}$ & $\begin{array}{r}14,5 \\
445\end{array}$ & $\begin{array}{l}55,{ }^{\prime} t \\
58^{\prime} t\end{array}$ & $\begin{array}{l}66,7 \\
44,4\end{array}$ & $\begin{array}{l}77,2 \\
5,7\end{array}$ & $\begin{array}{c}8 x^{\prime}, 8 \\
x^{\prime} y^{\prime} y^{\prime}\end{array}$ & $\begin{array}{l}56,6 \\
128\end{array}$ \\
\hline $\begin{array}{l}\text { I1. Orge-Levure sulf. } \\
\text { Poids moyen } \mathrm{kg} \\
\text { G.Mi.Q. en } \mathrm{g} . . .\end{array}$ & $\begin{array}{c}31,7 \\
2 \cdot 2 \cdot 2\end{array}$ & $\begin{array}{l}35,5 \\
978\end{array}$ & $\begin{array}{rl} & 33,8 \\
4 & 45\end{array}$ & $\begin{array}{c}53,7 \\
555\end{array}$ & $\begin{array}{c}64,3 \\
500\end{array}$ & $\begin{array}{c}74,1 \\
500\end{array}$ & $\begin{array}{l}83,7 \\
472\end{array}$ & $\begin{array}{l}55,: 3 \\
121\end{array}$ \\
\hline $\begin{array}{l}\text { III. Orge-Lactosérum } \\
\text { levuré : } \\
\text { Poids moyen } \mathrm{kg} . \\
\text { G.M.Q. en } \mathrm{g} \text {.... }\end{array}$ & $\begin{array}{r}31,7 \\
250\end{array}$ & $\begin{array}{l}35,6 \\
-78\end{array}$ & $\begin{array}{c}43,8 \\
500\end{array}$ & $\begin{array}{c}53,0 \\
528\end{array}$ & $\begin{array}{c}6: 2,7 \\
333\end{array}$ & $\begin{array}{c}72,3 \\
500\end{array}$ & $\begin{array}{l}81,0 \\
555\end{array}$ & $\begin{array}{l}54, t^{\prime} \\
4 \geq 0\end{array}$ \\
\hline
\end{tabular}

Signification statistique : $\mathrm{P} \leqslant 0,01: * * \quad \mathrm{P} \leqslant 0,05: *$

Régime - ; Périodes - ; Interaction Période $\times$ Régime $^{* *}$; Séries $* *$.

Durant les périodes de collecte, la vitesse de croissance a été la même avec les trois régimes. Cependant, au cours de la cinquième période, le gain moyen quotidien a diminué pour tous les animaux, sans cause apparente, et de façon variable suivant les régimes; cette diminution étant particulièrement importante pour celui contenant le lactosérum levuré. Ceci explique que l'interaction entre l'effet des régimes et 
celui des périodes est significative alors que les résultats sont semblables au cours des autres périodes.

\section{Digestibilité de la matière organique et de l'azote}

Les valeurs moyennes du coefficient d'utilisation digestive apparent de la matière organique (CUDMO) obtenues pour chacun des régimes au cours de 7 périodes de récolte sont peu différentes : la digestibilité du régime soja $(82,2$ p. Ioo) est légèrement supérieure à celle du régime lactosérum levuré $(80,9 \mathrm{p}$. Ioo) et inférieure à celle du régime levure sulfitique $(83,0 \mathrm{p}$. I00) ; mais ces différences ne sont pas significatives (tabl. 6).

On observe une augmentation de la digestibilité de la matière organique en fonction de l'âge (fig. I) ; puis elle se stabilise pour les régimes soja et lactosérum levuré à partir de la troisième période de récolte quand les animaux atteignent environ

TABI,EAU 6

CUD apparent de la matière organique $p .100$

\begin{tabular}{|c|c|c|c|c|c|c|c|c|}
\hline Période & 1 & 2 & 3 & i & 5 & 6 & 7 & Moyenne \\
\hline I. Orge-Soja ...... & 79,8 & 82,3 & $8: 2,9$ & $x: 1,6$ & 82,5 & 83,0 & $8:, 0$ & $8: 2$ \\
\hline II. Orge-Lev. sulf. .. & 79,2 & 80,5 & 81,3 & $8: \underline{-18}$ & 87,2 & $8 \mathbf{k}^{\prime}, 8$ & 85,5 & 83,0 \\
\hline $\begin{array}{l}\text { III. Orge-Lactosérum } \\
\text { levuré............... }\end{array}$ & 80,0 & 81,0 & 82,0 & 80,5 & 80,4 & 80,8 & 81,6 & 80,9 \\
\hline $\begin{array}{l}\text { Signification statistiqu } \\
\text { Régime - ; Périod } \\
\text { C.L.D.M.o. = }\end{array}$ & $\begin{array}{l}: \mathrm{P} \\
* * ; \\
\text {. inge } \\
\frac{\mathrm{M}}{\mathrm{M}}\end{array}$ & $\begin{array}{l}01:{ }^{0} \\
\text { ractio } \\
-\quad \text { li. } \\
\text { gérée }\end{array}$ & $\begin{array}{l}P \leqslant \\
\text { ériode } \\
\text { fécale } \\
\end{array}$ & $\begin{array}{l}5: * \\
\text { Régime } \\
100\end{array}$ & Série & - & & \\
\hline
\end{tabular}

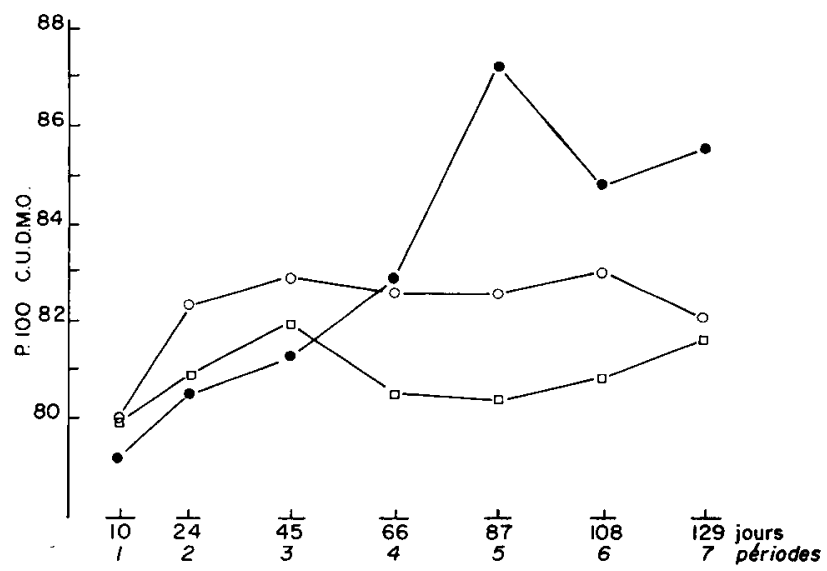

Fig. I. - Evolution du CUDMO en fonction de l'âge

- Régime orge-soja

a - — -lactosérum levuré

- — — - -levure sulfitique 
$50 \mathrm{~kg}$ de poids vif. Par contre, ce critère continue à augmenter avec le régime levure sulfitique.

Les valeurs moyennes du coefficient d'utilisation digestive apparent de l'azote (CUDN), pour l'ensemble des 7 périodes dans les trois régimes étudiés sont comparables : 8I,9 p. roo pour le régime soja ; 83,o p. Ioo pour le régime levure sulfitique et 83 , I p. Ioo pour le régime lactosérum levuré (tabl. 7). De même, l'évolution de ce critère avec l'âge est semblable pour les trois lots (fig. 2) : il augmente jusqu'à la troisième période de récolte, pour se stabiliser ensuite. De plus, la digestibilité de l'azote du lactosérum levuré est légèrement supérieure à celle du soja jusqu'à $54 \mathrm{~kg}$ de poids vif, alors que l'inverse a été observé dans le cas de la matière organique.

Contrairement à ce qui a été constaté pour la digestibilité de la matière organique, il n'y a pas d'interaction entre l'effet du régime et celui des périodes.

TABLEAU 7

CUD. apparent de l'azole p. 100
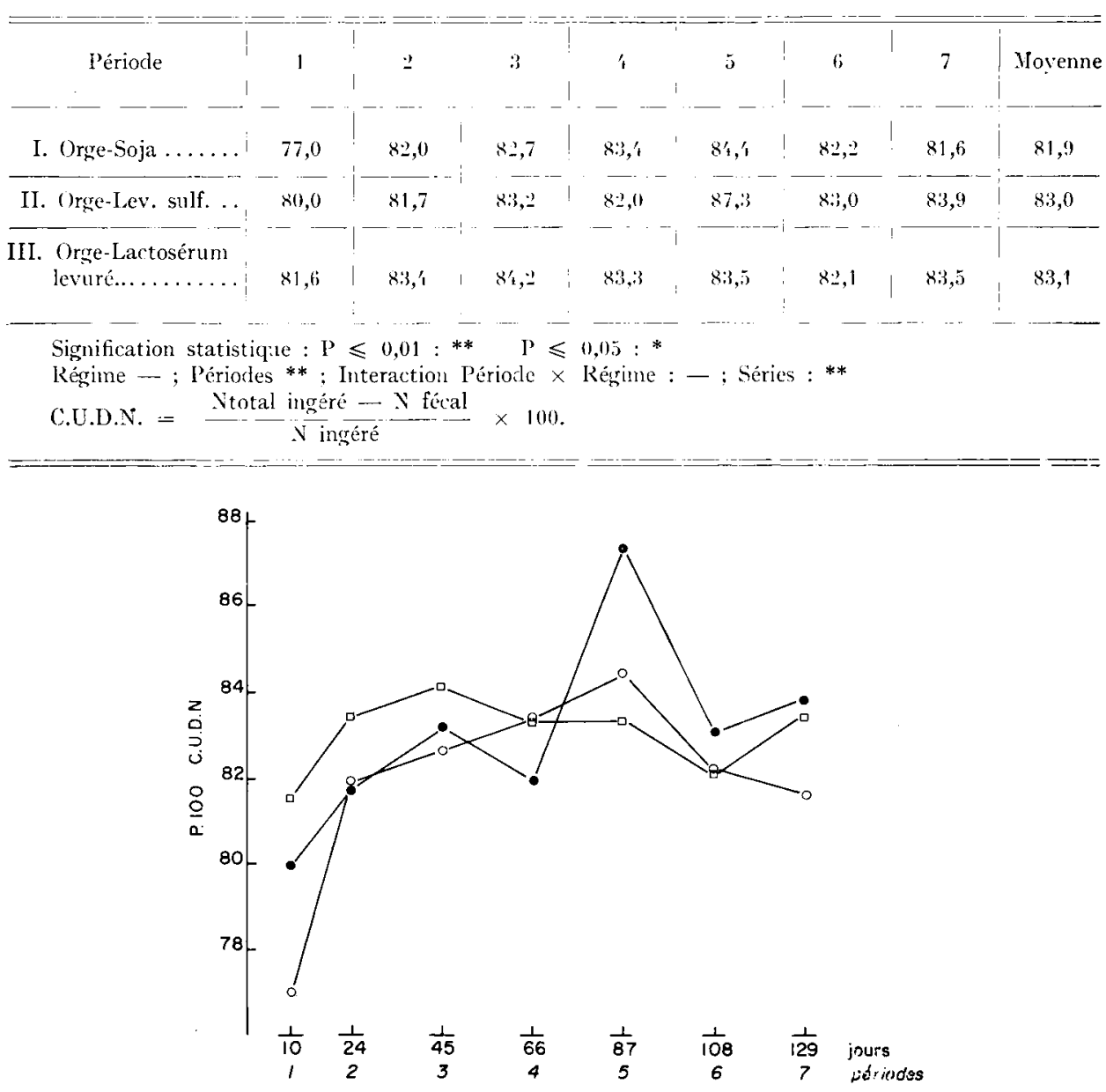

FIG. 2. - Évolution du CUDN en fonction de l'âge

○ Régime orge-soja $\square$ Régime orge-lactosérum levuré • Régime orge-levure sulfitique 


\section{Rétention azotée}

Il n'y a aucune différence en ce qui concerne la digestibilité de l'azote entre les trois régimes. En revanche, l'association orge-soja a permis une rétention quotidienne moyenne d'azote de $13,6 \mathrm{~g}$ pour l'ensemble des périodes, significativement plus élevée que celles obtenues avec les deux autres régimes : II, 6 g pour la levure sulfitique et II,7 g pour le lactosérum levuré (tab1. 8). L’ingestion de ces deux derniers a entrainé

\section{TABLEAU 8}

Azote retenu en g/jour

\begin{tabular}{c|c|c|c|c|c|c|c|c}
\hline Période & 1 & 2 & 3 & 1 & 5 & 6 & 7 & Ioyenne \\
\hline I. Orge-Soja ...... & 8,5 & 11,8 & 15,0 & 16,9 & 16,9 & 12,8 & 13,5 & 13,6 \\
\hline \begin{tabular}{c} 
II. Orge-Lev. sulf.... \\
\hline $\begin{array}{r}\text { II. Orge-Lactosérum } \\
\text { levuré.. ........ }\end{array}$
\end{tabular} & 8,5 & 11,2 & 12,2 & $1,2,9$ & 12,8 & 10,8 & 12,7 & 11,6 \\
\hline
\end{tabular}

Signification statistique : $P \leqslant 0,01: * * \quad P \leqslant 0,05: *$

Régime *; Périodes ** ; Interaction Période $\times$ Régime : — ; Séries : - .

une perte d'azote urinaire plus importante. Au cours de la croissance, le dépôt d'azote passe par un maximum dans la cinquième période de récolte, lorsque les animaux atteignent environ $60 \mathrm{~kg}$ de poids vif ; ceci est valable pour les trois régimes (fig. 3). Au-delà de ce poids, la quantité d'azote retenue diminue relativement moins rapidement pour le régime levure sulfitique que pour les deux autres régimes.

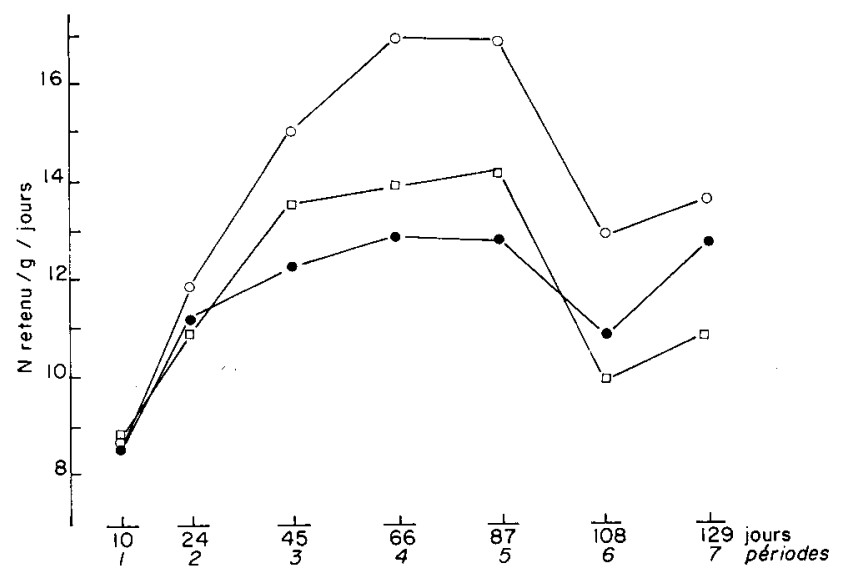

FIG. 3. - Évolution de $N$ retenu en gljour en fonction de l'âge

- Régime orge-soja $\square$ Régime orge-lactosérum levuré • Régime orge-levure sulfitique 
Corrélativement à un dépôt d'azote élevé, le coefficient de rétention de l'azote (CRN) obtenu avec le régime orge-soja est supérieur de $7 \mathrm{p}$. Ioo à celui constaté avec les deux autres régimes pour lesquels les résultats ne sont pas significativement différents (tabl. 9).

TABLEAU 9

Coefficient de rétention de l'azote, p. 100

\begin{tabular}{|c|c|c|c|c|c|c|c|c|}
\hline Période & 1 & 2 & 3 & 't & 5 & 6 & 7 & Moyenne \\
\hline $\begin{array}{c}\text { Régime }: \\
\text { Orge-Soja } \ldots \ldots \ldots\end{array}$ & 57,8 & 61,7 & 61,0 & 55,0 & 48,1 & 38,3 & 37,9 & 51,1 \\
\hline Orge-Levure sulfitique & 56,5 & 58,4 & 49,5 & 42,5 & 35,6 & 30,6 & 35,9 & 41,2 \\
\hline $\begin{array}{l}\text { Orge-Lactosérum } \\
\text { levuré } \ldots \ldots \ldots \ldots \ldots\end{array}$ & 56,0 & 55,3 & 53,9 & 41,4 & 41,8 & 29,1 & 31,3 & $1 \mathbf{t}_{1}, 5$ \\
\hline
\end{tabular}

Signification statistique : $\mathrm{P} \leqslant 0,01: * * ; \quad \mathrm{P} \leqslant 0,05: *$

Régimes ** ; Périodes ** ; Interaction période $\times$ Régrime —, Séries -

$\mathrm{CRN}=\frac{\mathrm{N} \text { ingéré }-(\mathrm{N} \text { fécal }-\mathrm{N} \text { urinaire })}{\mathrm{N} \text { ingéré }-\mathrm{N} \text { fécal }} \times 100$

soit : $\frac{N \text { retenu }}{N \text { absorbé }} \times 100$.

Au cours de la croissance, le coefficient de rétention de l'azote diminue à partir de la deuxième récolte (fig. 4) et vers $40 \mathrm{~kg}$ de poids vif pour les trois régimes. Cette diminution peut être attribuée au fait que le niveau d'alimentation est demeuré faible, et que les protéines ont été futilisées comme source énergétique, bien que le rapport Énergie/Azote des régimes employés soit relativement élevé en comparaison des besoins des animaux en croissance.

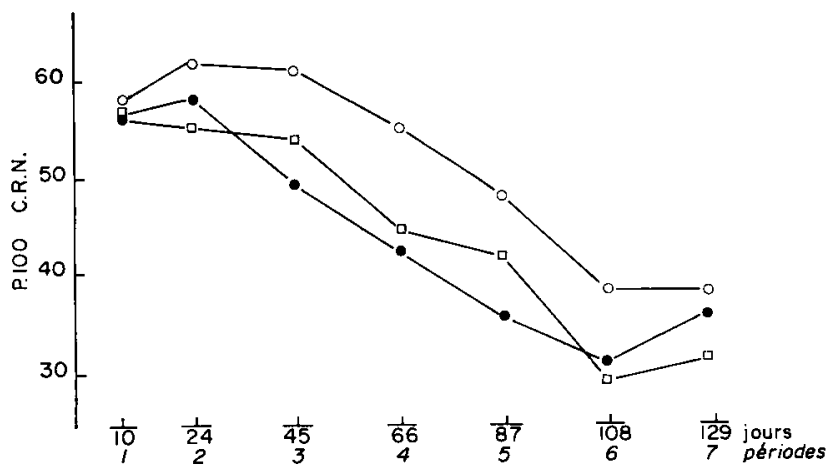

○ Régime orge-soja $\square$ Régime orge-lactosérum levuré $\bullet$ Régime orge-levure sulfitique

Le coefficient d'utilisation pratique de l'azote (CUPN) (fig. 5), évolue de la même manière que le coefficient de rétention puisqu'il est égal au produit CUDN $\times$ CRN et que les CUDN ne sont pas différents entre eux. 
Bien que la quantité d'azote retenue par jour soit supérieure avec le soja, et que le gain moyen quotidien pendant les périodes de collecte soit similaire entre les trois régimes, les différences observées en ce qui concerne la proportion d'azote retenue dans le gain de poids ne sont pas significatives (tabl. Io).

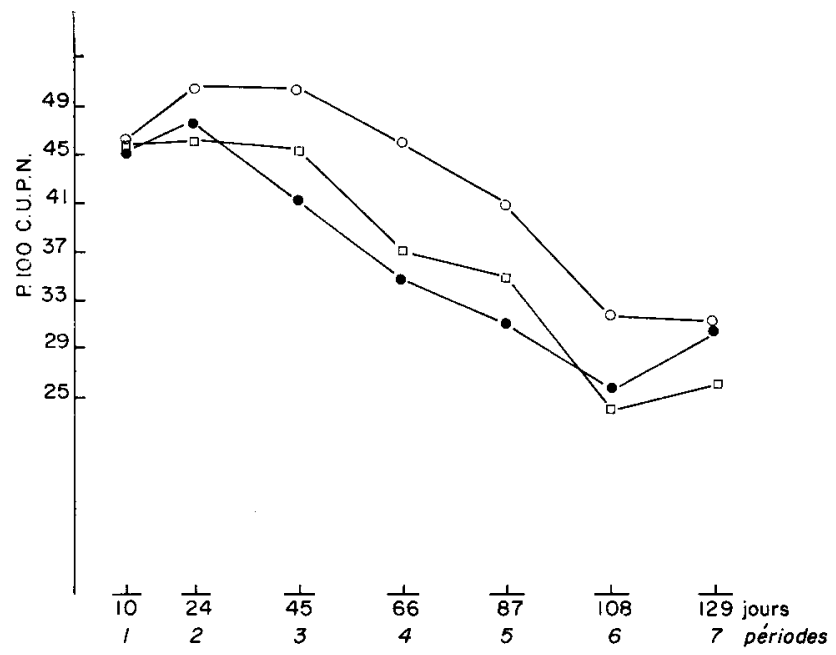

FIG. 5. - Evolution du CUP de $N$ en fonction de l'âge

o Régime orge-soja $\square$ Régime orge-lactorérum levuré - Régime orge-levure sulfitique

TABLEAU IO

Azole retenu en p. 100 du gain de poids

\begin{tabular}{c|c|c|c|c|c|c|c|c}
\hline \multicolumn{1}{|c|}{ Période } & 1 & 2 & 3 & 4 & 5 & 6 & 7 & Moyenne \\
\hline $\begin{array}{c}\text { Régime : } \\
\text { Orge-Soja ........ }\end{array}$ & 4,4 & 3,5 & 3,4 & 3,0 & 3,8 & 2,9 & 3,1 & 3,4 \\
\hline $\begin{array}{c}\text { Régime : } \\
\text { Orge-Lev. sulfitique .. }\end{array}$ & 4,2 & 4,0 & 3,1 & 2,3 & 2,5 & 2,2 & 2,7 & 3,0 \\
\hline $\begin{array}{c}\text { Régime : } \\
\text { Orge-Lact. Lev...... }\end{array}$ & 3,5 & 4,1 & 2,8 & 2,7 & 4,3 & 2,1 & 2,0 & 3,1 \\
\hline
\end{tabular}

Signification statistique : $\mathrm{P} \leqslant 0,01: * * \quad \mathrm{P} \leqslant 0,05: *$

Régimes -, Périodes $* *$; Interaction périodes $x$ régime -, Séries -

La proportion d'azote retenue dans la carcasse diminue avec l'âge, et de la même manière avec les trois régimes. 


\section{Résultats généraux de 30 à $90 \mathrm{~kg}$}

L'étude des résultats d'engraissement entre 30 et $90 \mathrm{~kg}$ comprenant des périodes durant lesquelles il n'y a pas eu de collecte, nous apporte quelques données complémentaires (tabl. II).

\section{TABLEAU II}

Résultats généraux entre 30 et $90 \mathrm{~kg}$ de poids aif

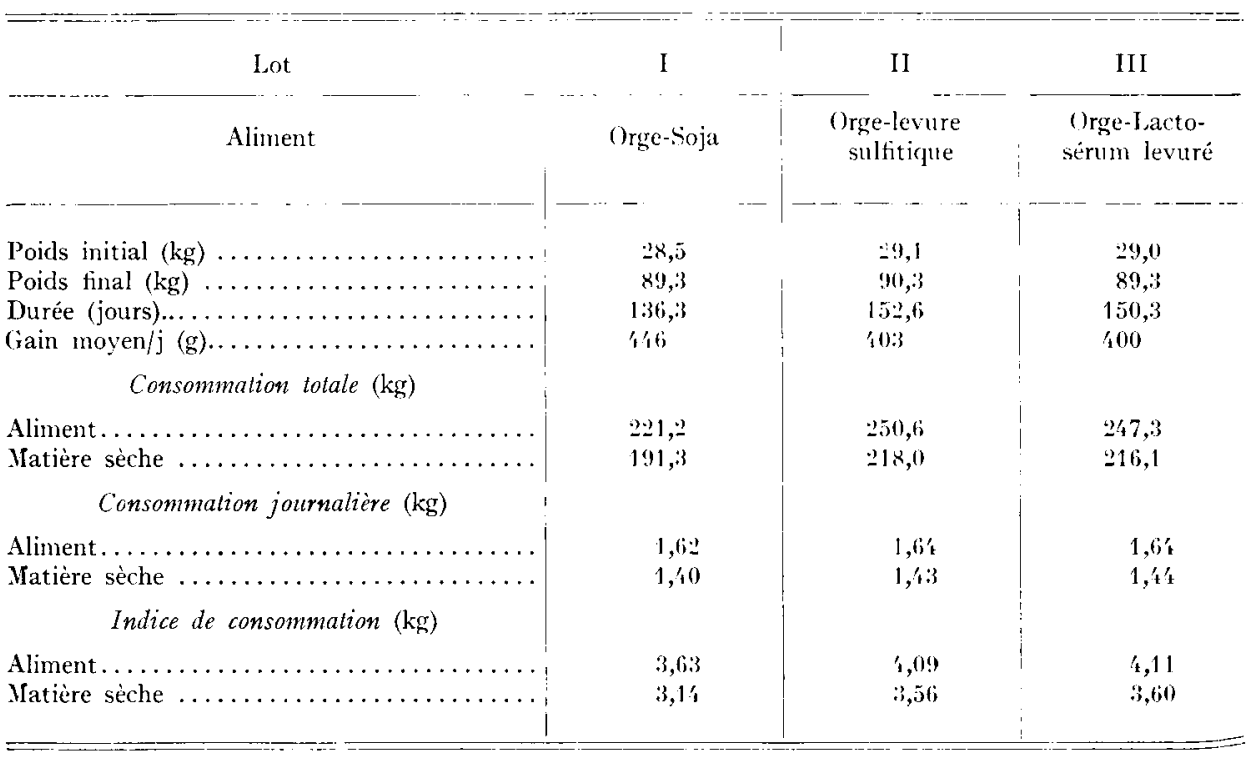

Sur l'ensemble de l'expérience, la vitesse de croissance des animaux du lot soja a été légèrement supérieure à celle des autres lots, mais la différence n'est pas significative, à cause du très petit nombre d'animaux mis en expérience.

La consommation d'aliment est pey différente entre les trois régimes puisque la plus grande partie de l'expérience a été réalisée en alimentation égalisée ; cependant, la consommation totale est légèrement inférieure avec le lot soja, la durée d'engraissement ayant été plus courte. L'indice de consommation correspondant est donc également inférieur à celui obtenu avec les autres régimes, mais les différences ne sont pas significatives.

\section{Composition corporelle}

Les carcasses obtenues avec le régime orge-soja sont nettement moins grasses que celles fournies par les deux autres régimes et notamment par la levure sulfitique (tabl. I2). Les différences, bien que n'étant pas significatives, compte tenu du petit nombre d'animaux, vont dans le même sens que celles concernant la rétention azotée 
déterminée par la méthode des bilans. La levure sulfitique a entraîné une rétention de protéines plus faible, et donc, à poids égal, la formation de carcasses plus riches en lipides.

\section{TABLEAU I2}

Résultats concernant la composition corporelle

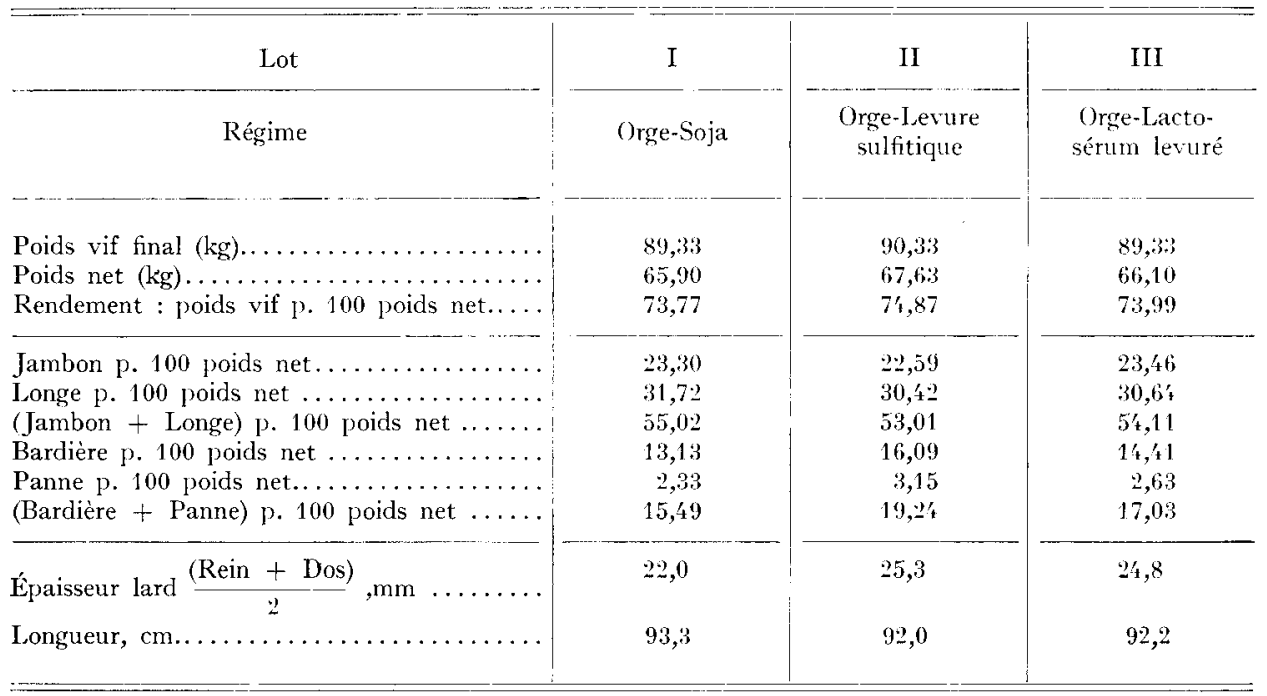

\section{DISCUSSION}

Vitesse de croissance des animaux et indice de consommation

On remarquera que si la vitesse de croissance est identique durant les périodes de collecte, elle est, pour l'ensemble de l'expérience, légèrement supérieure avec le régime soja. Ceci est dû en partie, au fait que la consommation a été laissée ad libitum après la dernière période de collecte et que ce régime a fourni d'une part une rétention azotée plus élevée, et d'autre part, un léger avantage en consommation quotidienne, donc en vitesse de croissance.

En valeur absolue, les gains moyens quotidiens sont inférieurs à ceux obtenus par Glaps et Ruszczyc (I955) ; mais il faut tenir compte du fait que dans notre expérience, les animaux étaient rationnés jusqu'à $83 \mathrm{~kg}$ de poids vif et maintenus en cage de digestibilité pendant toute la durée de l'engraissement.

Étant donné que la vitesse de croissance est faible, et par conséquent, la durée de l'engraissement plus longue, la dépense d'entretien totale des animaux est plus élevée, ce qui provoque une élévation de l'indice de consommation. Pour ces mêmes raisons, les indices que nous avons constatés sont supérieurs à ceux que les auteurs cités ci-dessus ont obtenus. 


\section{Digestibilité de la matière organique et de l'azote}

Compte tenu du protocole expérimental choisi, consistant à utiliser des régimes isoazotés, isocellulosiques, isoénergétiques, la partie du régime complémentaire de l'aliment à étudier n'a pas la même composition en ce qui concerne les teneurs en cellulose et en énergie ; il n'est donc pas possible de calculer la valeur exacte de la digestibilité de la matière organique de la levure sulfitique ou du lactosérum levuré ; on ne peut donc considérer que la rétention azotée.

Un résultat est cependant surprenant; il concerne 1'augmentation de la digestibilité de la matière organique de la levure sulfitique au cours de la croissance, phénomène qui ne s'observe pas avec les deux autres régimes. Il faut peut-être voir là une adaptation progressive de la flore intestinale du Porc à utiliser cette levure. Ce point serait à confirmer et à expliquer car il ne semble pas avoir encore été signalé chez cette espèce.

La digestibilité moyenne des protéines au cours de la croissance est équivalente pour les trois régimes. Étant donné que la proportion des protéines d'orge et des protéines à étudier est la même pour tous les régimes, on peut en conclure que les protéines de lá levure sulfitique, du lactosérum levuré et du soja ont une digestibilité moyenne équivalente.

Cependant, au cours des trois premières périodes, le CUD de l'azote du régime orge-lactosérum levuré est supérieur à celui du régime orge-soja, ensuite l'ordre est inversé. Ceci peut être interprété par la présence, dans le lactosérum, de la lactalbumine et de la lactoglobuline, protéines de haute digestibilité, qui sont absentes des deux autres régimes, et par la présence de lactose qui est moins bien utilisé en période de finition qu'en période de croissance.

\section{Rétention de l'azote}

La rétention de l'azote diminue avec l'âge : cette observation est en accord avec les travaux concernant l'étude du besoin azoté chez le Porc (RÉRAT et HENRY, I964), mais ce qui est très intéressant à noter, c'est la différence dansles coefficients de retention del'azote entre le régime orge-soja ( $5 \mathrm{I}, 4 \mathrm{p}$. I00) et ceux des régimes orge-levure et orgelactosérum levuré $(44,2$ p. Ioo et 44,5 p. Ioo respectivement). Ceci est confirmé par les différences significatives de gain d'azote en $g$ par jour et par les résultats concernant la composition corporelle. Quand on compare l'évolution du CRN avec l'âge, on constate une chute brutale à partir de la $2^{\mathrm{e}}$ période de récolte, vers $40 \mathrm{~kg}$ de poids vif, que l'on peut expliquer par l'apport insuffisant d'énergie dû à une consommation journalière d'aliment très faible. Cette faible ingestion entraînerait une utilisation de la source azotée du régime à des fins énergétiques, et par conséquent une croissance très réduite.

Lorsque 1'on compare les résultats concernant la quantité d'azote retenue en p. Ioo du gain de poids, donnés au tableau Io, on s'aperçoit que les différences obtenues avec les trois régimes ne sont pas significatives, ce qui n'est pas en accord avec les résultats concernant le Coefficient de Rétention de l'Azote et le gain azoté par jour. D'autre part, l'azote retenu en $\mathrm{p}$. Ioo du gain de poids pour les deux premières collectes semble être trop élevé. D'après nos résultats, l'azote déposé dans le gain de 
poids ne correspond pas à la composition corporelle des animaux qui contient en moyenne $3 \mathrm{p}$. roo d'azote, soit I9 $\mathrm{p}$. Ioo de matières azotées totales avec des variations de 15 à $20 \mathrm{p}$. Ioo. Cette erreur expérimentale peut être attribuée au fait que le gain de poids a été relativement faible durant chacune des périodes, et à l'imprécision de la pesée effectuée avec un pèse-bétail sur des porcs prenant en moyenne 2 à $3 \mathrm{~kg}$ par semaine. Par ailleurs, en règle générale, les bilans donnent des valeurs surestimées par rapport à l'analyse de la carcasse du fait des pertes dans les urines et les fèces.

Les résultats concernant le CRN et l'azote retenu par jour et ceux de la composition corporelle, dans leur ensemble, nous démontrent que les protéines du soja, dans le cas de la supplémentation de l'orge, et sans apport d'acides aminés, sont mieux utilisés que celles de la levure sulfitique ou du lactosérum levuré. Cette différence d'utilisation doit être expliquée par les différences de teneurs en acides aminés indispensables ou de leur disponibilité.

Quand on compare le pourcentage de déficit en aminoacides des régimes orge-soja et orge-levure sulfitique par rapport aux besoins calculés par RÉRAT et LoUGNON (I966,) (tabl. I3), on peut logiquement expliquer les différences de rétention azotée.

TABLEAU I3

Déficit des régimes par rapport aux besoins des porcs en croissance finition (en p. I०0)

\begin{tabular}{|c|c|c|c|}
\hline Régime & $\begin{array}{c}\text { I } \\
\text { Orge-Soja } \\
(\%)\end{array}$ & $\underset{\substack{\text { Orge-Lev. sulf. } \\
(\%)}}{\text { OI }}$ & $\begin{array}{c}\text { III } \\
(\%)\end{array}$ \\
\hline Acide aminé limitant & & & \\
\hline $\begin{array}{l}\text { 1.) de } 20 \text { à } 60 \mathrm{~kg} \\
\text { Iysine............. }\end{array}$ & 18 & 13 & 7 \\
\hline Acides aminés soufrés & 23 & 31 & 18 \\
\hline Isoleucine $\ldots \ldots \ldots \ldots$ & 10 & 18 & 7 \\
\hline Thréonine........... & 14 & 12 & - \\
\hline $\begin{array}{l}\text { 2.) de } 60 \text { à } 100 \mathrm{~kg} \\
\text { Acides aminés soufrés. }\end{array}$ & - & 9 & - \\
\hline
\end{tabular}

En effet, l'acide aminé limitant primaire est représenté, pour les deux régimes, par la somme des acides aminés soufrés (Méthionine plus Cystine), et ce déficit est plus important pour la levure sulfitique que pour le soja. Pendant la période de finition, seul le régime contenant la levure sulfitique présente encore ce déficit. Il est donc normal que l'on trouve, avec le mélange des protéines d'orge et de levure sulfitique, un coefficient de rétention de l'azote inférieur à celui obtenu avec l'association des protéines du tourteau de soja et d'orge.

Au contraire, lorsque l'on compare les résultats expérimentaux avec les compositions en acides aminés des régimes orge-soja et orge-lactosérum levuré, ce raisonnement n'est plus valable. En effet, le pourcentage de déficit en acides aminés soufrés 
est plus faible avec le lactosérum levuré qu'avec le soja et pourtant le coefficient de rétention de l'azote est supérieur avec ce dernier. Il faut donc admettre un déficit plus important de certains acides aminés qui concerne, soit les soufrés, soit d'autres aminoacides. On peut penser, en particulier, à une indisponibilité de la lysine due à un phénomène chimique tel que la réaction de Maillard entre la lysine et le lactose lors de la dessiccation du lactosérum levuré. Si cette hypothèse est exacte, il faut admettre un déficit en lysine supérieur à $I 8 \mathrm{p}$. Ioo par rapport aux besoins, valeur obtenue pour le régime orge-soja. Or, ce pourcentage de déficit entraîne un blocage de $82 \mathrm{~g}$ de lysine dans le lactosérum levuré (tabl. I4), ce qui représente une indisponibilité de 5 p. Ioo de celle-ci. Ceci est confirmé par la comparaison des résultats obtenus avec le lactosérum levuré et la levure sulfitique, résultats qui sont identiques. Comme le déficit en lysine et en acides aminés soufrés dans le dernier est plus important, cela montre qu'il y a une indisponibilité de la lysine encore plus importante dans le lactosérum levuré.

TABLEAU 14

Apport en lysine pour les éléments de base aux régimes expérimentaux $I$ et $I I$, exprimé en grammes

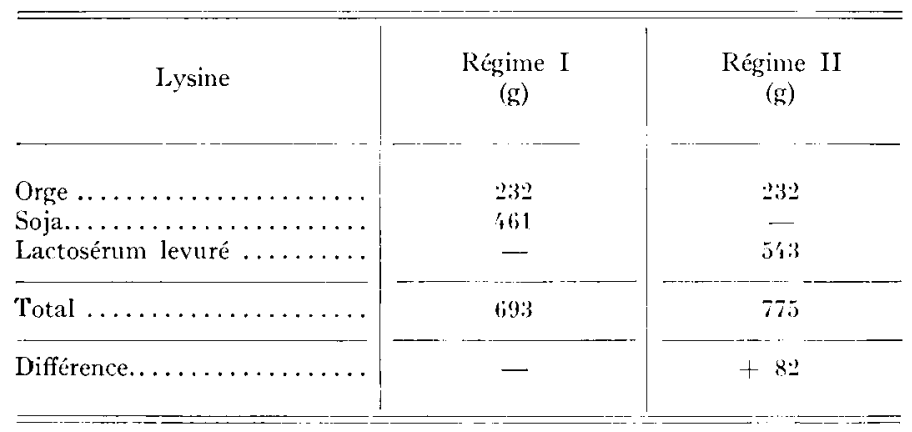

Ces résultats concordent avec ceux obtenus par CALE'T et al., r962, qui montrent que la supplémentation de la levure de bière par la méthionine dans un régine à base de céréales n'a aucun effet chez le Rat, la lysine étant le facteur limitant. Le même type de résultats a été obtenu par Rous et al. (1965) chez le Poulet et par NAvRaTII. et al. (I966) chez le Porc entre $30-5^{\circ} \mathrm{kg}$ avec des régimes à base de céréales; une addition de méthionine n'a d'effet que dans le cas où il y a également supplémentation de lysine.

\section{Composition corporelle}

Corrélativement à une rétention azotée plus élevée, les carcasses obtenues avec le régime orge-soja sont plus maigres que celles obtenues avec les deux autres régimes. Le petit nombre d'animaux considérés ne permet pas de donner une signification statistique à cette différence : néanmoins, elle va dans le même sens que la plupart des observations faites sur l'influence des levures sur l'augmentation de l'adiposité des carcasses lorsqu'on les substitue à des protéines de haute qualité, (Ruszczyc et et GlaPS, I955; NAVRatil, et al., I966). 
Par contre, ces résultats sont contraires à ceux obtenus par Bokorof et al., (I963), qui obtiennent des carcasses plus grasses, avec du lait écrémé et de la farine de poisson qu'avec de la levure. Cependant, comme la proportion de levure était de $5 \mathrm{p}$. Ioo en période de croissance et de $\mathrm{I}, 9 \mathrm{p}$. Ioo en période de finition, on peut se demander si la levure a eu réellement une grande influence par elle-même. Ces résultats sont toutefois à rapprocher de ceux obtenus par FÉvRIER et BoCCARD (I957) qui notent une amélioration avec $4 \mathrm{p}$. Ioo de levures dans le régime, mais aucun avantage pour des taux plus élevés puisqu'avec $36 \mathrm{p}$. Ioo de la ration, la vitesse de croissance est réduite. De même, les taux de levures que nous avons employés se situent au-dessus de la proportion optimum définie par CsoKa (I965) à 20 p. Ioo des protéines du régime.

La question est donc de savoir si ces résultats sont dus seulement à une indisponibilité de la lysine ou, éventuellement, à d'autres facteurs.

\section{CONCLUSION}

La comparaison de la valeur de supplémantation des protéines du soja, de levure sulfitiqu: et du lactosérum levuré vis-à-vis des protéines d'orge, nous a montré que la digestibilité de ces proténes n'était pas d:fférente, mais que, en revanche, le coefficient de rétention de 1'azote et l'azote retenu en g par jour étaient nettement plus élevés avec le soja qu'avec les levures. Ceci est confirmé par le fait que les carcasses obtenues sont plus grasses, bien que les croissances aient été sensiblement comparables avec les régimes levurés dans les trois lots. Cette différence ne peut pas s'expliquar uniquement sur la base des teneurs en acides aminés totaux des différents rézimes, en particulier pour le lactosérum levuré. L'hypothèse la plus plausible est que, outre une déficience en acides aminés soufrés, il existe une indisponibilité de $1 x$ lysine due à un traitement thermique in approprié ou à une autre cause actuellement indéterminée.

Reçu pour publication en septembre 1968.

\section{SUMMARY}

FEEDING VALUE OF PROTEINS OF SULPHITE YEAST AND YEAST-TREATED WHEY FOR GROWING PIGS

Digestibility and retention of nitrogen of sulphite yeast grown on residues of paper pulp, of whey treated with yeast and of soyabean meal as supplements to barley were studied in an experiment with three groups of three pigs between 30 and $90 \mathrm{~kg}$ liveweight.

Diets were similar in protein content, in energy value and in fibre content, and $\mathrm{Ca}$ and $\mathrm{P}$ content also were equalized (tables 3 and 4). Rationing was in relation to age; it was the same for all the animals and was adjusted according to the smallest intake observed.

Results obtained show that the mean values for apparent digestibility of organic matter did not differ significantly : 82.2 per cent for the diet of barley and soya, 83.0 per cent for that of barley and sulphite yeast and 80.9 per cent for that of barley and yeast-treated whey (table 3). One result was nevertheless surprising; it concerns the increase in digestibility of organic matter of sulphite yeast during growth, a phenomenon not observed with the other two diets (figure 1 ). 
Mean values for digestibility of nitrogen, combined for the seven periods, of the diets studied were comparable : 81.9 per cent for the diet of barley and soya, 83.0 per cent for that of barley and sulphite yeast and 83.I per cent for that of barley and yeast-treated whey (table 7). During the first three periods percentage digestibility of nitrogen of the diet with whey was higher than that of the diet with soya, and after that the order was reversed (fig. 2 ).

In contrast, the amount of nitrogen retained daily on the diet of barley and soya was $13.69 \mathrm{~g}$, significantly greater than the II.6 and Ir.7 $\mathrm{g}$ retained on the diets of barley and sulphite yeast and of barley and yeast-treated whey, respectively (table 8 and fig. 3). Correspondingly, retention of nitrogen of the diet of barley and soya bean meal, 5 r.4 par cent, was significantly greater than on the diet with sulphite yeast, 44.2 per cent of with yeast-treated whey, 44.5 per cent (table 9 and fig. 4). These results are confirmed by the body composition of the pigs after cutting of the carcasses. The pigs fed on the diet of barley and soya ware leaner than those on the other two diets (table 12). These results suggest that the protein of soya, as a supplement to barley are more suitable to cover the amino acid requirements of pigs from 40 to $90 \mathrm{~kg}$ than the protein of the yeast products used.

If the results with the diet of barley and sulphite yeast are in accord with amino acid composition of this diet, the same is not true as regards the diet of barley and yeast-treated whey in which the deficit in essential amino acids in less than seen in the diet of barley and soyabean meal (table 13). This contradiction could be explained by accepting the hypothesis of the existence of a lack of availability of lysine due to some chemical phenomenon, such as the Maillard reaction, between lysine and lactose during drying of the yeast-treated whey. According to our calculations this lack of availability would be of the order of 15 per cent (table 14 ).

\section{RÉFÉRENCES BIBLIOGRAPHIQUES}

Bokorov T., Delic I., SREckovic A., 1963. Comparison of the biological value of fodder yeast ((Torula utilis), dried milk and fishmeal as additives to gluten feed and soya bean oilmeal for fattening pigs (serbocroate). Stocarsivo, 17, 560-565.

Calet C., Adrian J., Jacquot R., I962. Étude de la valeur nutritionnelle de la levure utilisée sous trois formes différentes : Levure sèche, levure autolysée et autolysat de levure. Ann. Zootech., 11, Io3-I I4.

Cochran W. G., Cox G. M., 1957. Experimental designs, 6i i pp. Ed. John Wiley and sons. New York.

CsoKa S., 1965. Effect of different amounts of dried brewer's yeast in feed mixtures on the fattening performance of Hungarian Yorkshire pigs fed from self-feeders (hong.) Allattenyesztes, 14, 55-66.

DaN I. R., Lakatos S., I959. The nutritive value of fodder yeast, Torulopsis utilis (roumain). Lucr. Inst. Cercel. Zoot., 3, I69-1 77 .

Fevrier R., Boccard R., I957. Valeur alimentiure des levures. V. La levure séchée sur marc de pommes. Ann. Zootech., 6, 269-28I.

Glaps J., Ruszczyc $Z_{\text {, }}$ 1955. Studies on the use of fodder yeast and maize in the fattening of pigs (pol.). Rocz. Nauk. Rol. (B), ro, I39-1 52.

HENRY Y., RÉrat A., I966. Utilisation des pommes de terre déshydratées et fraîches dans l'alimentation du Porc en croissance en comparaison avec l'orge. Ann. Zoolech., 15, 231-251.

ISAJEV F., 1965. Dietetic and nutritional effects of yeast on weight gains of pigs and egg production of hens (tchèque). Zivoc. Vyr., 1, 31-40.

JACQUOT R., 1946. Valeur alimentaire de quelques protides de remplacement. Bull. Soc. Hyg. Aliment., 20, $645^{-660 .}$

LfFrançois L., Revuz B., I964. Levures de lessives sulfitiques. Description détaillée d'une réalisation récente. Industr. Alim., Agr., 421, I I 75-1 I8I.

Leroy A. M., Fevrier R., 1947. Emploi de la levure de distillerie pour l'alimentation des animaux domestiques. Ann. agron. Paris, 4, 578-596.

Naiditch V., I96i. Procédé de fermentation de lactosérum sans traitement préalable, Obtention d'un aliment levuré complexe. Industr. Alim. Agr., 3, 285-288.

Navratil B.; Simencek K., 1966. Comparison of the effectiveness of dried feed yeasts supplemented with L-Lysine and DL-Methionine and fish meal in the fattening of pigs (tchèque), Zivoc. Vyr 11, 17I-I82.

Palamaru E., Gondos M., Geaman N., Sirbu M., Petrovszky R., Romanovchi S., Hagea I., Covaliu C., ERdei M., Maxim V., Stavri J., ig66. Efficacité de la levure fourragère administrée dans la nourriture des jeunes porcs et des jeunes volailles (roumain). Lucr. Inst. Cercel. Zoot., 13, 185-201.

Pion R., i967. Données non publiées.

Pion R., Fauconneau G., 1966. Les acides aminés des protéines alimentaires. Méthodes de dosage et résultats obtenus. Cahier A.E.C., 6, I $55^{-1} 75$. 
Rérat A., Henry Y., ig64. Étude du besoin azoté chez le Porc en croissance. I. Utilisation de la farine de poisson à trois taux différents. Ann. Zootech., 13, 5-34.

RÉrat A., Lougnon J., ig66. Les besoins en amino-acides du Porc en croissance. Cahier A.E. C., 6, 34I-422.

Rous J., Petkovs S., Stefk M., Skrivan M., 1965. Dried fodder yeast (Torulopsis utilis) and DL-Methionine in feed rations for chicks (tchèque). Zivoc. Vyr., 10, 749-758.

Ruszczyc Z., Glaps J., 1955. a) The use of post-sulphite yeast in the fattening of pigs (Pol.). Rocz. Nauk. Rol. (B), 70, 169-180.

Ruszczyc Z., Glaps J., 1955. b) The use of yeast slop (Fresh brewery yeast) for fattening pigs (pol.). Rocz. Naul. Rol. (B), 70, $153-167$.

Ruszczyc Z., Glaps J., r959. The feeding value of yeast produced on post-sulphite lyes and post-sulphite mash for fattening pig (pol.) Rocz. Nauk. Rol., (B), 74, I37-144.

Salmon-Legagneur E., ig6o. Les préférences alimentaires du porcelet. IV. Appétibilité des levures. Ann. Zootech., 9, 255-260. 